\title{
Achromatic Elemental Mapping Beyond the Nanoscale in the Transmission Electron Microscope
}

\author{
K. W. Urban* \\ Peter Grünberg Institute and Ernst Ruska Centre for Microscopy and Spectroscopy with Electrons (ER-C), \\ Research Centre Jülich, D-52425 Jülich, Germany \\ J. Mayer \\ Gemeinschaftslabor für Elektronenmikroskopie and ER-C, RWTH Aachen University, Ahornstrasse 55, \\ D-52074 Aachen, Germany \\ J. R. Jinschek \\ FEI Company, Achtseweg Noord 5, 5651 GG Eindhoven, Netherlands \\ M. J. Neish, N. R. Lugg, and L. J. Allen \\ School of Physics, University of Melbourne, Parkville, Victoria 3010, Australia
}

(Received 18 January 2013; published 2 May 2013)

\begin{abstract}
Newly developed achromatic electron optics allows the use of wide energy windows and makes feasible energy-filtered transmission electron microscopy (EFTEM) at atomic resolution. In this Letter we present EFTEM images formed using electrons that have undergone a silicon $L_{2,3}$ core-shell energy loss, exhibiting a resolution in EFTEM of $1.35 \AA$ A. This permits elemental mapping beyond the nanoscale provided that quantum mechanical calculations from first principles are done in tandem with the experiment to understand the physical information encoded in the images.
\end{abstract}

DOI: 10.1103/PhysRevLett.110.185507

PACS numbers: 61.05.jd, 68.37.Lp, 68.37.Og

Energy-filtered transmission electron microscopy (EFTEM) is a technique that images a specimen using inelastically scattered electrons that have undergone a specific range of energy losses within the specimen [1-3]. By selecting energy windows that cover a range of energies pertinent to inner-shell ionization of elements present in the sample, it is possible to obtain chemical maps and bonding information of different atomic species. This is possible over a large field of view, acquiring the structural information in parallel (as opposed to serially in scanning transmission electron microscopy, STEM).

However, up to now it was not possible to realize atomic resolution in EFTEM. Chromatic aberration degrades the image formed, as electrons that have lost different amounts of energy within an energy window will be focused in different image planes. This effect can be reduced by decreasing the width of the energy window. However, this also leads to a reduction of the signal to noise ratio. Because of these competing effects, the resolution of energy-filtered images has been limited to about $4 \AA$ $[4,5]$. Recently chromatic aberration $\left(C_{\mathrm{C}}\right)$ correction has been implemented [6] to supplement the now ubiquitous spherical aberration $\left(C_{\mathrm{S}}\right)$ correctors in electron optics $[7,8]$. We demonstrate here that this allows wide energy windows to be used and atomic resolution to be realized. This enables transmission electron microscopy to combine the atomically-resolving picometer-precision interferometric imaging with atomic-resolution chemical information.
The FEI Titan 60-300 PICO at the Ernst Ruska Centre is a fourth-generation transmission electron microscope capable of obtaining high-resolution transmission electron microscopy images approaching $50 \mathrm{pm}$ resolution in the $C_{\mathrm{C}^{-}}$and $C_{\mathrm{S}^{-}}$-corrected mode at $300 \mathrm{keV}$. It is currently one of only two microscopes in the world capable of chromatic aberration correction. Here we present the first experimental EFTEM images using the $C_{\mathrm{C}^{-}}$and $C_{\mathrm{S}}$-corrected optics for a sample of crystalline silicon. Quantum mechanical calculations from first principles were performed and are an essential adjunct to elucidating the process of electron propagation and image formation.

Experimental EFTEM images of silicon were obtained using signal from the $L_{2,3}$ ionization edge (threshold energy for ionization $\sim 100 \mathrm{eV}$ ). The microscope was operated at $300 \mathrm{keV}$ with a semi-convergence angle for the incident beam of $1.7 \mathrm{mrad}$. The specimen, a wedge whose thickness varied between 100 and $400 \AA$, was imaged along the [110] zone axis. The three-window technique [3] was used with the pre-edge images centered at energy losses of 55 [Fig. 1(a)] and $75 \mathrm{eV}$ [Fig. 1(b)] to do a background subtraction on the raw post-edge data, for the energy window centered at $120 \mathrm{eV}$ [Fig. 1(c)], thus obtaining the Si elemental map shown in Fig. 1(d). Energy slit widths of $40 \mathrm{eV}$ were used, which was only possible using the achromatic objective lens $\left(C_{\mathrm{C}}=0\right)$. Overlapping windows were chosen in the pre-edge region in order to avoid positioning the first pre-edge window in the strongly 


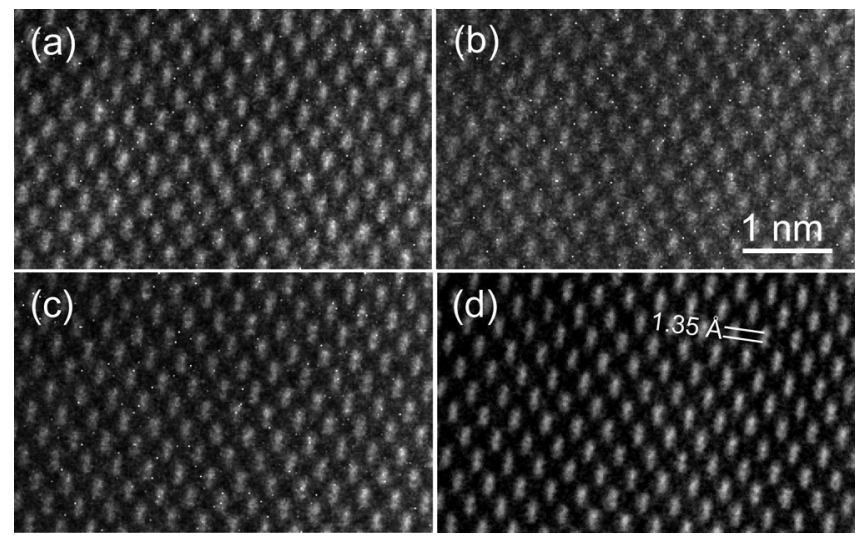

FIG. 1. EFTEM images used to construct the Si $L_{2,3}$ elemental map by means of the three-window technique in the chromaticand spherical-aberration corrected electron microscope. All images were taken using a $40 \mathrm{eV}$ wide energy window. (a) Pre-edge image centered at an energy loss of $55 \mathrm{eV}$. (b) Pre-edge image centered at $75 \mathrm{eV}$. (c) Post-edge image centered at $120 \mathrm{eV}$. (d) Resulting atomic-resolution elemental map; a standard "average background subtraction filter" [23] was applied. Standard conditions for the negative-spherical aberration imaging mode $[19,20]$ were applied in all images, i.e., a (negative) $C_{\mathrm{S}}$ of $-8.27 \mu \mathrm{m}$ and a defocus of $+40 \AA$.

oscillating plasmon-loss region. The atomic "dumbbell" structure of $\mathrm{Si}$ in [110] projection is resolved, where the centers of the two atoms in each dumbbell pair are separated by $1.35 \AA$.

To understand the complex physics and electron optics underpinning the formation of the elemental map in Fig. 1(d) it is necessary to model the elastic and inelastic scattering of the incident electrons in the specimen. To do this we supplement the approach in Ref. [9] with the use of the quantum excitation of phonons (QEP) model [10], which calculates the underlying elastic and thermal diffuse scattering of the incident electrons and provides the basis for then modeling the ionization of the silicon atoms by both elastically and thermally scattered electrons. In the context of the QEP model the intensity in the recording plane (the EFTEM image) is given by

$$
I\left(\boldsymbol{r}_{\perp}\right)=\int \sum_{\alpha, n}\left|T\left(\boldsymbol{r}_{\perp}\right) \otimes \phi_{\alpha, n}\left(\boldsymbol{r}_{\perp}, t, \boldsymbol{\tau}\right)\right|^{2}\left|a_{0}(\boldsymbol{\tau})\right|^{2} d \boldsymbol{\tau} .
$$

Here, $T\left(\boldsymbol{r}_{\perp}\right)$ is the transfer function of the imaging lens which is convolved with the auxiliary functions $\phi_{\alpha, n}\left(\boldsymbol{r}_{\perp}, t, \boldsymbol{\tau}\right)[10]$ associated with the electron at the exit surface of a specimen of thickness $t$ (after ionization of atom $\alpha$ to leave the system in a final state $n$ ). We consider final states that are consistent with the acceptance range of the energy filter. The integration is a quantum mechanical average over nuclear coordinates where $\left|a_{0}(\tau)\right|^{2}$, the modulus squared of the wave function describing the nuclear subsystem for the set of nuclear coordinates $\tau$, is acting as a probability distribution and accounts for the crystal being in a superposition of phonon states. We note that Eq. (1) is consistent with different ionization events being incoherent with respect to each other.

If the atom $\alpha$ is at a depth $z$ in the specimen then the auxiliary function at the exit surface, $\phi_{\alpha, n}\left(\boldsymbol{r}_{\perp}, t, \boldsymbol{\tau}\right)$ in Eq. (1), is obtained after channeling the auxiliary function generated at $z$, immediately after ionization, through a distance $t-z$ to the exit surface. The generation of the auxiliary function at $z$ due to an ionization event is given by $[10,11]$

$\phi_{\alpha, n}\left(\boldsymbol{r}_{\perp}, z, \boldsymbol{\tau}\right)=\frac{m}{2 \pi i h^{2} k_{n}} H_{\alpha, n 0}\left(\boldsymbol{r}_{\perp}, \boldsymbol{\tau}\right) \phi_{0}\left(\boldsymbol{r}_{\perp}, z, \boldsymbol{\tau}\right)$.

Here, $m$ is the relativistically corrected electron mass, $h$ is Planck's constant, and $k_{n}$ is the wave number of the electron after inelastic scattering (also relativistically corrected). The modulus squared of the transition potential $H_{\alpha, n 0}\left(\boldsymbol{r}_{\perp}, \boldsymbol{\tau}\right)$ for an excitation of atom $\alpha$ from the initial (bound) state 0 to the final (continuum) state $n$ gives the probability of that transition occurring. The $\tau$ dependence indicates that the potential moves with the atom for each new configuration. The function $\phi_{0}\left(\boldsymbol{r}_{\perp}, z, \boldsymbol{\tau}\right)$ associated with the electron prior to ionization is obtained by channeling the incident wave from the entrance surface for the configuration of atoms specified by $\boldsymbol{\tau}$. We use an angular momentum basis to represent the transition potentials, the details of which are described elsewhere [12]. Although it is convenient to think about each inelastic transition (associated with ionization) separately, a formulation in terms of density matrices is also possible [13].
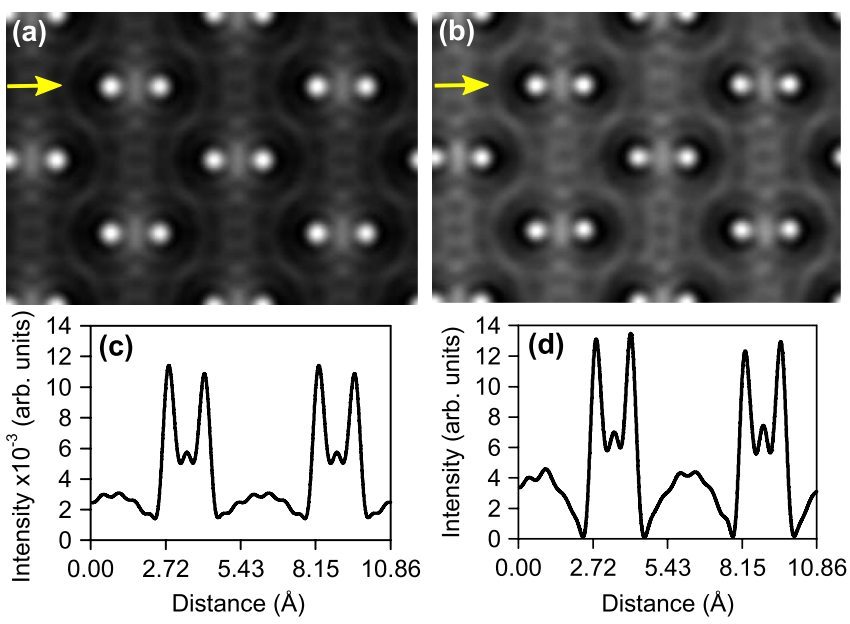

FIG. 2 (color online). Calculations of EFTEM images formed using the silicon $L_{2,3}$ edge for a $200 \AA$ thick specimen imaged at $300 \mathrm{keV}$ with a beam convergence angle of $1.7 \mathrm{mrad}$. (a) EFTEM image formed due to ionization by both elastically and thermally scattered electrons in the QEP model [10] and (b) an image formed by electrons which have only been elastically and thermally scattered. Line scans across the images in (a) and (b) taken through the dumbbell pairs (as indicated by the arrows) are shown in (c) and (d), respectively. 
Calculations were carried out to simulate the experimental results in Fig. 1(d). We assumed a $200 \AA$ thick specimen, a (negative) $C_{\mathrm{S}}$ of $-8.27 \mu \mathrm{m}$ and a large objective aperture size of $2 \AA^{-1}$ (consistent with experiment). Assuming a defocus value of $+40 \AA$ yields the result shown in Fig. 2(a). It is interesting to note that there is significant intensity between the atomic positions. This could explain the intensity between the columns in the dumbbells in Fig. 1(d). Figure 2(b) shows an image formed directly by electrons which have only been elastically and thermally scattered. Line scans across the images in Figs. 2(a) and 2(b) were taken along the dumbbell pairs, as indicated in Figs. 2(a) and 2(b) and are shown in Figs. 2(c) and 2(d), respectively. We see that the contrast is similar. As we have verified, elastic scattering dominates the contrast in Fig. 2(b), with the contribution from thermally scattered electrons being small. This is an example of the phenomenon usually termed "preservation of elastic contrast" [14-17].
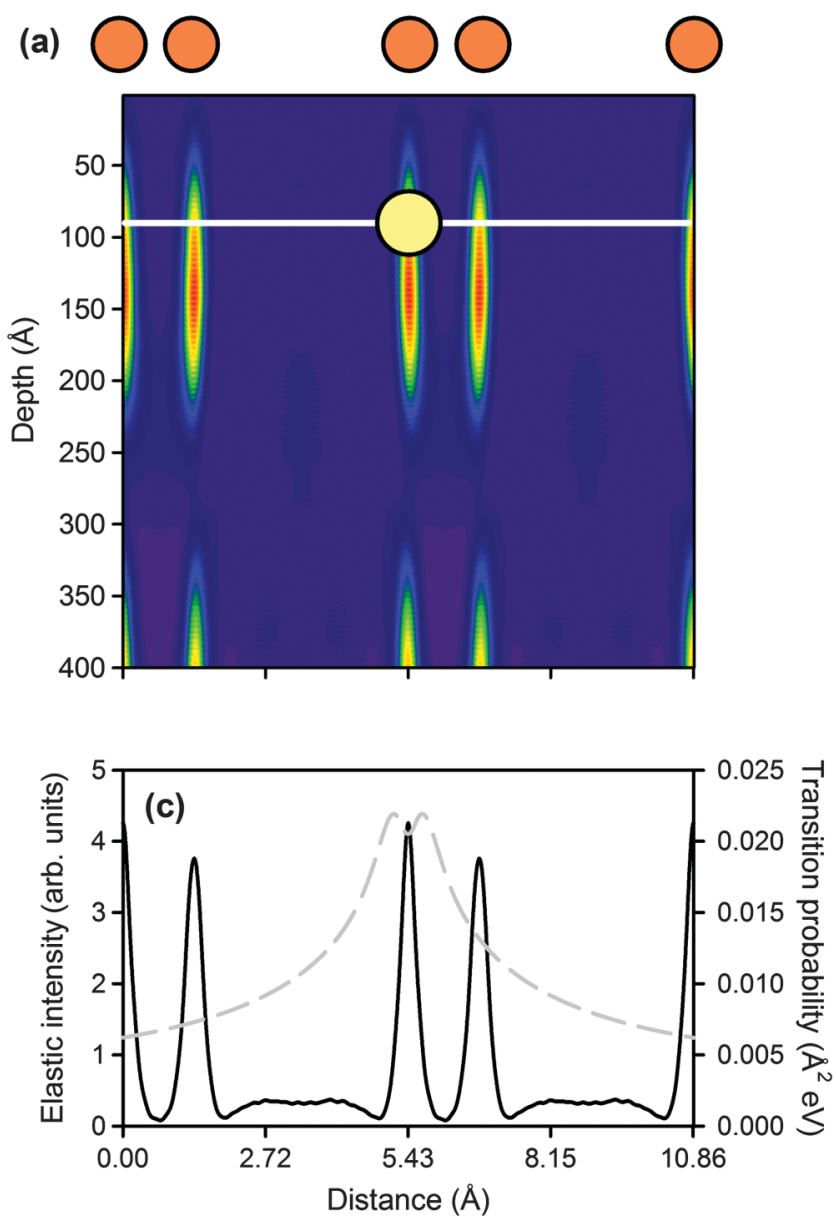

From our transition potential approach, we can explain why the images in Figs. 2(a) and 2(b) look qualitatively similar. For high-energy incident electrons causing ionization, the transition potentials become more extended ("delocalized") the lower the energy loss. As a consequence features of the elastic and thermal scattering prior to and after the ionization event contribute directly and significantly to the energy-filtered image. Indeed, from Eq. (2) we can see that, for a relatively wide transition potential, features (amplitude and phase) in the wave function of the incident electrons $\psi_{0}$ away from the column being ionized will be preserved in the inelastic wave function $\psi_{n}$ [18]. This is evident in the experimental preedge and post-edge images where lattice contrast is observed in both, since the contribution to these images is mainly due to delocalized inelastic scattering leading to the excitation of plasmons. Figure 3(a) shows the intensity of the elastic wave function, along a line scan in the [001] direction, horizontally through the dumbbell structure, as it propagates through the sample. The intensity of the elastic
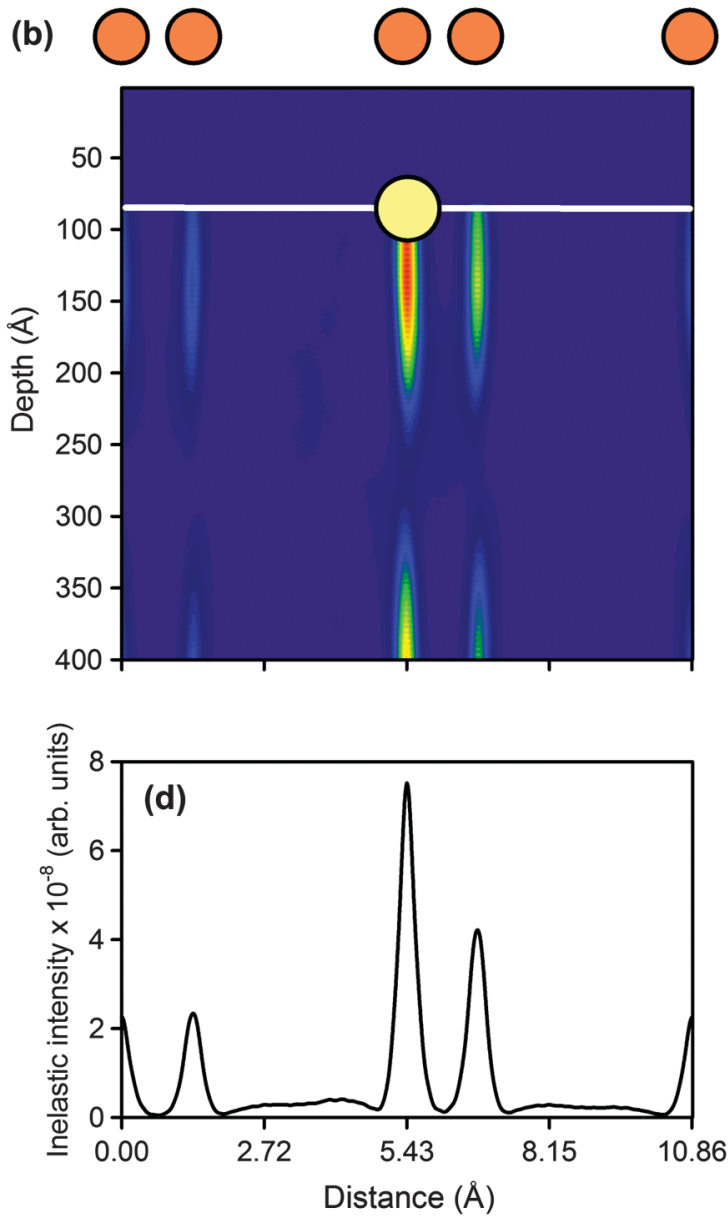

FIG. 3 (color). Probe intensity within silicon. Line scans are along the [001] direction. An ionization of a Si $L_{2,3}$ edge [specifically $\left(l=1, m_{l}=0\right) \rightarrow\left(l^{\prime}=0, m_{l^{\prime}}=0\right)$ transition] occurs at a depth of $80 \AA$. (a) Intensity along the [110] direction of the elastic wave as a function of depth. (b) Intensity along the [110] direction of the inelastic wave as a function of depth. (c) Intensity of the elastic wave function (black) and the transition probability $\left|H_{n 0}\right|^{2}$ (gray), at a depth of $80 \AA$. (d) Overlap of the elastic intensity and the transition potential. 


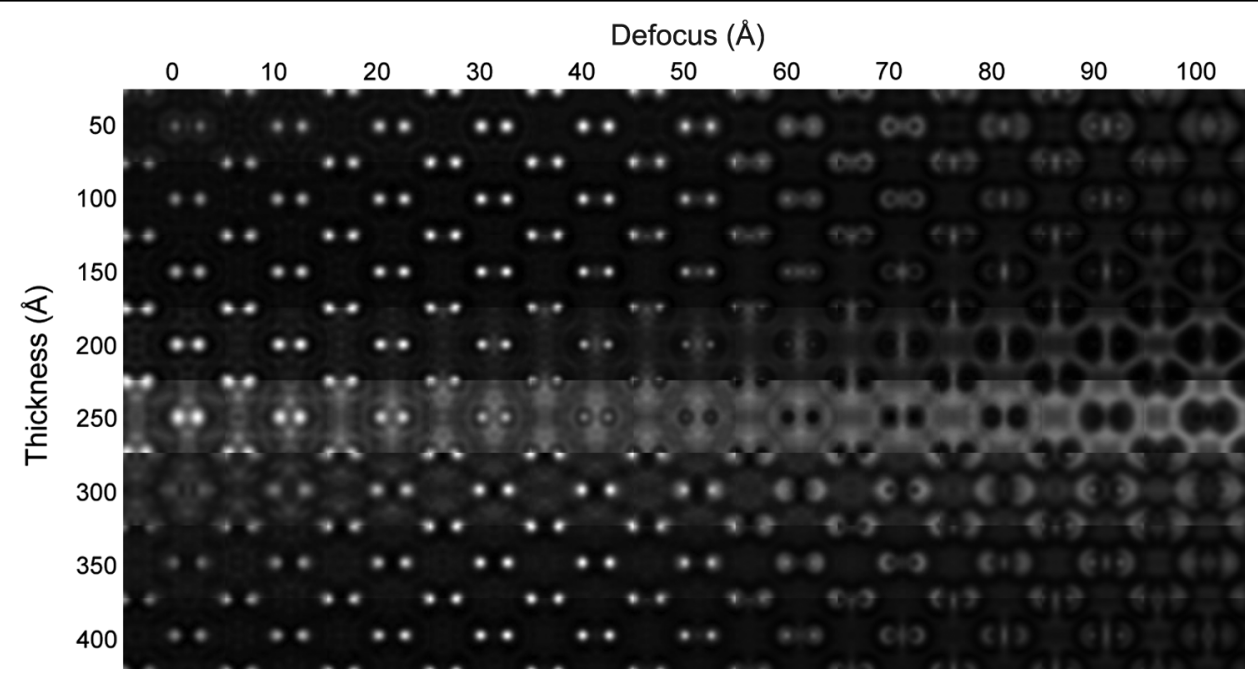

FIG. 4. Thickness-defocus series of EFTEM images for the Si $L_{2,3}$ edge at $300 \mathrm{keV}$. An objective lens with a (negative) $C_{\mathrm{S}}$ of $-8.27 \mu \mathrm{m}$, an objective aperture of $2 \AA^{-1}$ and varying defocus has been used. Each image has been normalized to the maximum value for a given thickness for visualization purposes.

wave is highest on the atomic columns, due to channeling through the specimen. We now consider an ionization event occurring at a depth of $80 \AA$ from the entrance surface. Specifically, we consider the $\left(l=1, m_{l}=0\right) \rightarrow$ $\left(l^{\prime}=0, m_{l^{\prime}}=0\right)$ dipole transition in the angular momentum basis used here. The inelastic wave function in Fig. 3(b) for this ionization also "illuminates" the other atom in the dumbbell pair, albeit with a lower intensity, which then channels to the exit surface. The intensity of the elastic wave function at the ionization event is presented in Fig. 3(c). The two peaks correspond to the atomic columns where the dumbbells sit. They are at slightly different heights as the atoms within each column are situated at different depths within the specimen. Overlaid is the transition probability $\left|H_{n 0}\right|^{2}$ for the specific ionization event. The transition probability is substantially delocalized and extends across the neighboring atomic columns. Figure 3(d) shows the intensity of the inelastic wave function generated at the ionization event, which is the product of the elastic wave function and the transition probability. As a result of the delocalized $H_{n 0}$, there is a significant signal on nearby columns.

The effects that the post-ionization elastic channeling has on the EFTEM image can be seen in the thicknessdefocus series of EFTEM images in Fig. 4. The series was calculated for thicknesses between 50 and $400 \AA$, and defocus values ranging between 0 and $+100 \AA$. It is found that the EFTEM images appear qualitatively similar at varying specimen depths, with the intensity varying with thickness due to the channeling of the incident electron probe. The lattice contrast is most visible between defocus values of 30 to $50 \AA$, consistent with predictions of negative spherical aberration imaging $[19,20]$. The similarity of the EFTEM images over the thickness range investigated is due to only being one element present in the specimen.
This was also observed in the experimental elemental map as one moves away from the edge of the $\mathrm{Si}$ wedge. However, one can still notice the effects of elastic contrast being preserved. In Fig. 3(a), the pendellosung of the elastic wave produces an intensity minimum at around $250 \AA$. Since at this thickness there is less elastic signal on the column being ionized, we should expect a large proportion of the ionization signal to come from points away from the column. This is verified in Fig. 4 where, for a specimen thickness of around $250 \AA$, we see that a large proportion of the signal forms a diffuse background, away from the column.

In conclusion, we have demonstrated experimentally that by using spherically and chromatically corrected electron optics the resolution of EFTEM can be improved to atomic as proven by the $1.35 \AA \mathrm{Si}$-dumbbell imaging. This allows unambiguous identification of the chemical nature of individual atom columns in the transmission electron microscope on the basis of images produced by electrons that have experienced a characteristic inner-shell excitation energy loss. To date this has been the domain of STEM $[21,22]$. From this point on it is feasible to combine the ultrahigh precision atomic position measurements on the basis of the interferometric imaging mode enabled by exitplane wave function reconstruction in the aberration corrected transmission electron microscope with chemical mapping, atom column by atom column in the same area on the sample. Our first-principles calculations show that the pertinent phenomena can be well described within a quantum mechanical model for elastic and inelastic scattering. The images obtained show the phenomenon of preservation of elastic contrast.

The financial support of the German Federal Ministry for Science and Education, the Ministry of Innovation of the State of North-Rhine Westphalia, the German Research 
Foundation (DFG) and the Helmholtz Association for the PICO project is gratefully acknowledged. This research was supported under the Discovery Projects funding scheme of the Australian Research Council (Project No. DP110102228). Special thanks are due to C. L. Jia and L. Jin for providing the silicon samples and for assistance with Digital Micrograph ${ }^{\mathrm{TM}}$.

*k.urban@fz-juelich.de

[1] R. F. Egerton, Electron Energy Loss Spectroscopy in the Electron Microscope (Plenum Press, New York, 1996).

[2] C.C. Ahn, Transmission Electron Energy Loss Spectrometry in Materials and the EELS Atlas (WileyVCH, Weinheim, 2004), 2nd ed.

[3] W. Jäger and J. Mayer, Ultramicroscopy 59, 33 (1995); F. Hofer and P. Warbichler, in Ref. [2], p. 159.

[4] B. Freitag and W. Mader, J. Microsc. 194, 42 (1999).

[5] T. Walter, Ultramicroscopy 96, 401 (2003).

[6] B. Kabius, P. Hartel, M. Haider, H. Müller, S. Uhlemann, U. Loebau, J. Zach, and H. Rose, J. Electron Microsc. 58, 147 (2009).

[7] M. Haider, S. Uhlemann, E. Schwan, H. Rose, B. Kabius, and K. Urban, Nature (London) 392, 768 (1998).

[8] M. Haider, P. Hartel, H. Müller, S. Uhlemann, and J. Zach, Phil. Trans. R. Soc. A 367, 3665 (2009).

[9] N. R. Lugg, B. Freitag, S. D. Findlay, and L. J. Allen, Ultramicroscopy 110, 981 (2010).
[10] B. D. Forbes, A. V. Martin, S. D. Findlay, A. J. D’Alfonso, and L. J. Allen, Phys. Rev. B 82, 104103 (2010).

[11] W. Coene and D. van Dyck, Ultramicroscopy 33, 261 (1990).

[12] S.D. Findlay, M. P. Oxley, and L. J. Allen, Microsc. Microanal. 14, 48 (2008).

[13] J. Verbeeck, P. Schattschneider, and A. Rosenauer, Ultramicroscopy 109, 350 (2009).

[14] A. Howie, Proc. R. Soc. A 271, 268 (1963).

[15] S. L. Cundy, A. Howie, and U. Valdrè, Philos. Mag. 20, 147 (1969).

[16] P. Stallknecht and H. Kohl, Ultramicroscopy 66, 261 (1996).

[17] T. Navidi-Kasmai and H. Kohl, Ultramicroscopy 81, 223 (2000).

[18] C. J. Humphreys and M. J. Whelan, Philos. Mag. 20, 165 (1969).

[19] C. L. Jia, M. Lentzen, and K. Urban, Science 299, 870 (2003).

[20] K. W. Urban, C. L. Jia, L. Houben, M. Lentzen, S. B. Mi, and K. Tillmann, Phil. Trans. R. Soc. A 367, 3735 (2009).

[21] D. A. Muller, L. Fitting Kourkoutis, M. Murfitt, J. H. Song, H. Y. Wang, J. Silcox, N. Dellby, and O.L. Krivanek, Science 319, 1073 (2008).

[22] L. J. Allen, S. D. Findlay, and M. P. Oxley, in Scanning Transmission Electron Microscopy, edited by S.J. Pennycook and P.D. Nellist (Springer, New York, 2011), p. 247.

[23] R. Kilaas, J. Microsc. 190, 45 (1998). 